\title{
High Resolution DOA Estimation technique for uplink TDD mode
}

\author{
P. Marques ${ }^{1,2}$, A. Gameiro ${ }^{1}$ and J. Fernandes ${ }^{1}$ \\ ${ }^{1}$ Universidade de Aveiro, Instituto de Telecomunicações, DETUA, 3810 Aveiro, Portugal \\ ${ }^{2}$ Escola Superior de Tecnologia - Instituto Politécnico de Castelo Branco, 6000 Castelo Branco, Portugal
}

\begin{abstract}
This paper investigates a maximum likelihood (ML) approach for direction of arrival (DOA) estimation based on the algorithm suggested by the 3GPP for scalar channels in UTRA-TDD mode. The performance of the algorithm is assessed by resorting to simulations in typical UMTS scenarios. The results indicate that the proposed scheme overcomes the limitation of the angular resolution inherent to classical techniques like subspace or beam-forming methods. In particular, it is shown that waves exhibiting an arbitrarily small difference in azimuth and temporal spacing higher than one chip time interval can be easily separated. A threshold operation is included to select only the most significant paths, in terms of energy, of the estimated channel. The results show that a good performance can be achieved from low to high values of $E_{b} / N_{0^{*}}$
\end{abstract}

\section{INTRODUCTION}

Future 3G-CDMA mobile radio communication systems have to respond to the increasing demand for transmission capacity. To meet this challenge, these systems may incorporate advanced signal processing techniques, such as the use of antenna arrays, but to achieve a good performance, accurate estimates of the DOA of each multipath component impinging on the antenna array is required.

In the UTRA-TDD mode, the burst structure specifies a specially designed midamble to allow channel estimation by cyclic correlation [1]. The channel estimation algorithms considered in this work are based on the standard one suggested by 3GPP for UTRA-TDD. Previous work have shown that the performance of this algorithm is highly related with the temporal spreading between consecutive paths [2]. To increase the time resolution we propose a Successive Multipath Estimation Technique (SMET) to replace the 3GPP scalar channel estimator.

Conventional DOA estimation methods are based on classical beamforming techniques and require a large number of array elements to achieve high resolution. Subspace based methods are high-resolution sub-optimal techniques which exploit the eigen structure of the input data matrix, e.g. MUSIC (Multiple Signal Classification) and ESPRIT (Estimation of Signal Parameters via Rotational Invariance Technique), but it degrades severely in a multipath propagation environment [3]. Maximum likelihood (ML) techniques are optimal and which perform well even under low signal-to-noise ratio conditions, typical of WCDMA systems, but are computationally very demanding. To overcome this limitation, we implemented a moderate complexity ML DOA

This work was partially funded by the European IST 199910741 ASILUM project. estimation based on SMET. The DOA algorithm proposed in this paper consists in applying the SMET for the scalar channel estimation to each of the array elements, and theh estimate the DOA using the ML criterion based on the phase shift relation existing between the array elements. In the case of a radio channel with $L$ multipath components and considering $K$ users, this algorithm reduces the ML problem to $K x L$ successive 3 -dimensional maximization problem. The estimation algorithms were implemented and integrated in a link level evaluation platform for the UTRA-TDD physical layer

The paper is organized as follows. Section II presents the conventional 3GPP channel estimation algorithm. Section III describes the SMET proposed and we made a time resolution and error analysis. In section IV, first the performance of subspacebased techniques for DOA estimation is analysed and next an extension of the SMET algorithm using the ML principle to estimate the DOA is proposed. The performance of the algorithm is assessed by evaluating the rms error of the DOA estimates. Finally the main conclusions of this work are presented in Section V.

\section{Il. CONVENTIONAL 3GPP CHANNEL ESTIMATION ALGORITHM}

In the UTRA-TDD mode, the burst structure specifies a specially designed midamble to allow channel estimation by cyclic correlation. The block diagram for the scalar channel estimation unit is shown in Figure 2. The midambles of different users active in the same time slot are time shifted versions of one single periodic basic code, $m p$. The specification document defines a $\mathrm{Lm}$ $=512$ chip midambles for Burst Type 1 and $L m=256$ chips midambles for Burst Type 2 [1]. Standard channel estimation algorithm for UTRA-TDD basically computes the correlation between the received midamble sequence and the locally generated $m p$ basic code. The different user specific channel impulse response estimates are obtained in a specific $W_{k}$-chip window as shown in Figure 1. The channel estimator in each $W_{k^{-}}$ chip window, search the $L$ dominant peaks. The locations and complex amplitudes of these peaks constitute the delay estimates, $\tau$, amplitude $\alpha$, and phase $\theta$ of the lt $h$ multipath component.

The following "two-wave" scenario is used as a basis for assessing the resolution ability of algorithm. Neglecting noise and the disturbance caused by non-ideal correlation the output of the correlator in the corresponding window be written as:

$$
W_{0}(t)=\alpha_{1} e^{j \theta_{1}} g\left(t-\tau_{1}\right)+\alpha_{2} e^{j \theta_{2}} g\left(t-\tau_{2}\right)
$$




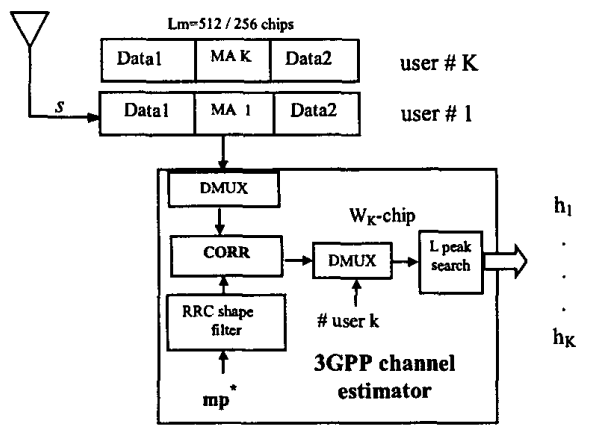

Fig.1. Conventional 3GPP channel estimation unit.

where $\alpha e^{j \theta}$ is the single complex fading coefficient, and $\mathrm{g}($. is the root raised cosine shaping filter with a roll off of 0.22 . The error estimation analysis of the coefficients and delays paths is made in the worst case, that is, two paths are in phase. Figure 2 shows the 3GPP channel estimation error plane. In this analysis, is considered an amplitude error criterion of $15 \%$. The number of samples per chip is 4 .

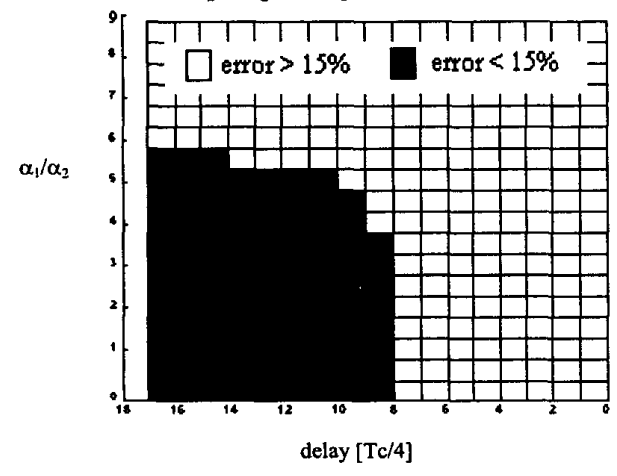

Fig.2. Conventional 3GPP channel estimation error plane (noise free).

Due to shape filter bandwidth limitation (W), the 3GPP scalar estimation algorithm can only resolve multipath components if they are separated by more than $\Delta t \approx 1 / W=2$ time chip (Tc), for UMTS that is $520 \mathrm{~ns}$. On the other hand, in the peak search process, the secondary lobes of the root raised cosine chip filter can be interpreted as false paths components, this happen if the amplitude ratio between two exceeds 5 . This situation can be see in Figure 3.

\section{SUCCESSIVE MULTIPATH ESTIMATION TEChNiQUE}

Measurements have shown that in Indoor and Pedestrian environments the temporal spacing between consecutive paths may be less than 520 ns [4]. To accommodate such scenarios the conventional $3 \mathrm{GPP}$ algorithm was modified to improve the time resolution. This technique we termed
SMET and includes one multipath discrimination process in the conventional scalar estimator.

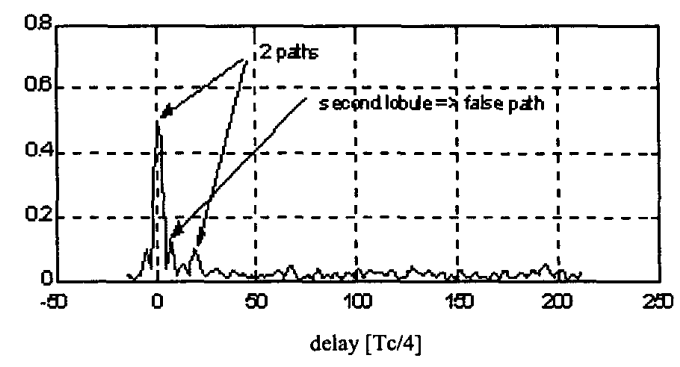

Fig.3. Typical window channel estimation ( $\left.\left|W_{0}\right|\right)$ : secondary lobe is interpreted as a false signal path.

The SMET consists in successively searching the highest peak of the window produced by the output correlator, and subtract to the overall waveform the elementary pulse with the parameters obtained from the highest peak. With such a scheme the accuracy of the multipath estimates improve at the output of the successive multipath cancellation stages. This technique has evident similarities with the soft decision successive interference cancellation (SIC) [5] used in multi-user detection. The SMET algorithm for two fingers consists in applying the following procedure:

$$
\begin{aligned}
& W_{0}(t)=3 G P P(\# k \text { user }) \quad \text { output estimator } \\
& W_{1}(t)=W_{0}(t) \\
& \text { for } i=1 \text { to Nstage } \\
& \quad \hat{\alpha}_{1}^{(i)}=\max \left|W_{1}\right| \\
& \quad\left(\hat{\theta}_{1}^{(i)}, \hat{\tau}_{1}^{(i)}\right)=\arg \max \left|W_{1}\right| \\
& \quad W_{2}(t)=W_{1}(t)-\hat{\alpha}_{1}^{(i)} e^{j \hat{\theta}_{1}^{(i)}} g\left(t-\hat{\tau}_{1}^{(i)}\right) \\
& \hat{\alpha}_{2}^{(i)}=\max \left|W_{2}\right| \\
& \left(\hat{\theta}_{2}^{(i)}, \hat{\tau}_{2}^{(i)}\right)=\arg \max \left|W_{2}\right| \\
& W_{1}(t)=W_{0}(t)-\hat{\alpha}_{2}^{(i)} e^{j \hat{\theta}_{2}^{(i)}} g\left(t-\hat{\tau}_{2}^{(i)}\right)
\end{aligned}
$$

Figure 4 shows the SMET error plane after two stages. In Figure 4 we can see that a significant improvement is achieved with SMET algorithm. This technique is able to resolve two multipath components with temporal spacing of 1 Tc. Simulations have shown a negligible error decrease when using more than two stages. This multipath discrimination technique has a low algorithmic complexity, and do not significantly increase the overall complexity of scalar channel estimator. 


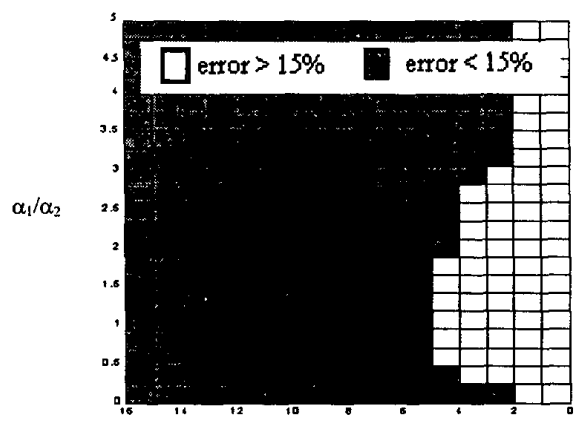

delay $[\mathrm{Tc} / 4]$

Fig.4. SMET error plane, Nstage $=2$ (noise free).

\section{A. Threshold level}

Figure 5-(a), clearly shows the existence of peaks that are caused by noise and interference, which could be interpreted as false paths contributing with additional interference in the receiver and consequently degrading the bit error rate. To minimize this problem the SMET incorporates a threshold operation in order to activate only the fingers with sufficiently high signal-tointerference plus noise ratio (SINR). After the SMET the total interference may be estimated by:

$$
\hat{n}(t)=W_{0}(t)-\hat{\alpha}_{1} e^{j \hat{\theta}_{1}} g\left(t-\hat{\tau}_{1}\right)-\hat{\alpha}_{2} e^{j \hat{\theta}_{2}} g\left(t-\hat{\tau}_{2}\right)
$$

$\hat{n}$ is caused by several disturbances: the additive white Gaussian noise, present at the antenna input, the intracell interference due to other users within one time slot, the intercell interference due to other cells with different $m p$ codes, and the estimation errors. Let us consider to make the analysis tractable that $\hat{n}$ is Gaussian distributed with variance $\sigma^{2}$. Defining a parameter $p$ as the tolerable probability for a false path, immediately defines the threshold as:

$$
T H=\sqrt{-2 \sigma^{2} \ln (p)}, \quad 0<p<1
$$

Figure 5 shows the sequence of threshold level computation. In this example the second path has a low SINR, thus it is eliminated by threshold processing.

\section{B. SINR estimation}

Power control is applied for the UTRA-TDD mode to limit the interference, thus, the burst structure specifies inclusion of a specially transmit power control (TPC) bits based on the SINR measurements [6]. Therefore, the channel estimation unit must to calculate the SINR. After SMET the SINR may be easy estimated by (4).

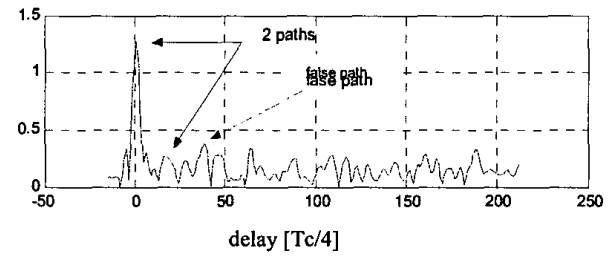

(a)

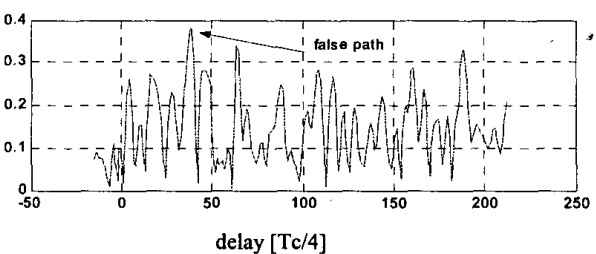

(b)

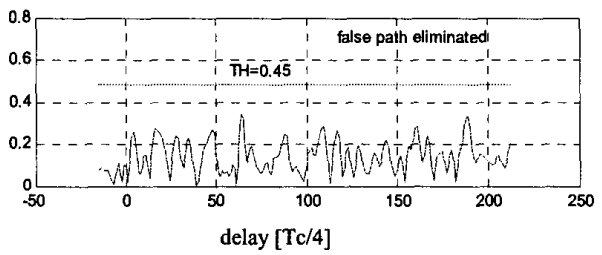

(c)

Fig.5. (a) $\left|W_{1}\right|$ : output 3GPP channel estimator, (b) $\left|W_{2}\right|$ : window after strong path elimination (c) $|n(\mathrm{t})|:$ residual interference plus noise term and the threshold level calculated with $p=1 \mathrm{E}-4$.

$$
S I N R=E\left[\frac{\sum_{i=1}^{L} \hat{\alpha}_{i}^{2}}{2 \sigma^{2} L m}\right]
$$

In [7] the SINR is computed across 160 UMTS slots, thus the fast variation due to fading can be sufficiently averaged.

\section{Simulation results with SMET}

The scalar channel estimation performance is examined in UTRA-TDD link-level simulation chain and implemented according to current 3GPP specifications [8]. To assess its performance, simulations have been carried out, using two uncorrelated paths with Rayleigh fading. The average power of the second path is $1 \mathrm{~dB}$ below the first path and the relative delay $(\Delta \tau)$ is 1 Tc. For this scenario, Figure 6 shows the mean power of the output estimation error (Perror), calculated by (5), for each path, using 3GPP scalar estimation algorithm and SMET.

$$
\operatorname{Perror}_{l}=E\left[\left|\alpha_{l} e^{j \theta_{l}}-\hat{\alpha}_{l} e^{j \hat{\theta}_{l}}\right|^{2}\right], \quad l=1,2
$$


It follows from Figure 6 that Perror is $3 \mathrm{~dB}$ lower if the SMET is applied, for $\mathrm{Eb} / \mathrm{N} 0=0 \mathrm{~dB}$, and this performance gain increases to $6 \mathrm{~dB}$ for $\mathrm{Eb} / \mathrm{N} 0=10 \mathrm{~dB}$. The effect of imperfect channel estimation on the overall link BER performance, for a scalar RAKE receiver, is shown in Figure 7. In this plot the uncoded BER obtained using the SMET is compared against the 3GPP estimator and the ideal case where the channel delays, amplitudes and phases are perfectly known. A spreading factor of the transmitted QPSK data symbols (SF) of 16 is used.

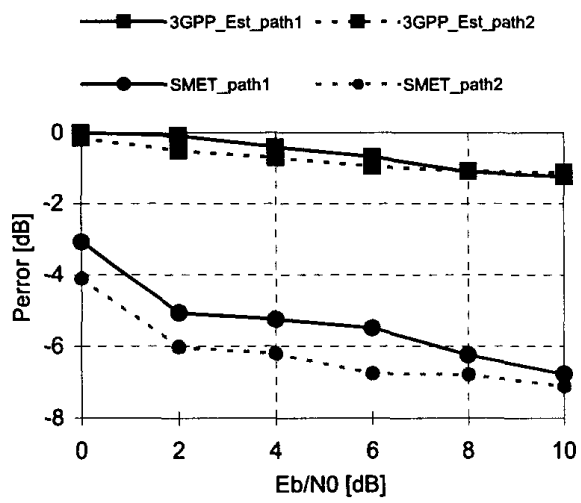

Fig.6. Comparison of the power estimation error in two Rayleigh paths scenario with $\Delta \tau=1 \mathrm{Tc}$.

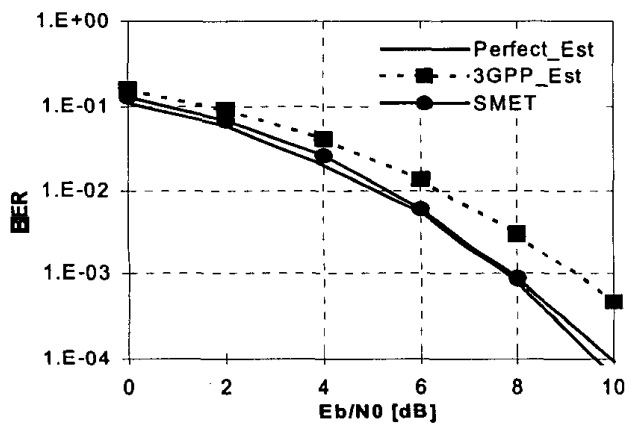

Fig.7. BER for 2-Rayleigh path channel, $\Delta \tau=1 \mathrm{Tc}$, Rake receiver with 2 fingers, $S F=16$, Burst Type 1 .

In Figure 7 we can see that SMET BER curve is very close to the lower bound achieved with perfect knowledge of channel parameters. The results show a significant performance improvement provided by the use of SMET, which brings along very low complexity increase compared to the standard technique.

\section{DOA ESTIMATION}

\section{A. Subspace-based techniques}

Various algorithms to estimate the DOA can be found in literature. The most popular uses subspace-based techniques to exploit the eigen structure of the input covariance matrix, for example MUSIC and ESPRIT. The most significant drawbacks of the subspace-based techniques are [3] [9]:

- These methods degrade severely in a multipath propagation environment, especially with low SINR.

- $M$ element array can only detect $M-1$ not correlated signals.

- Require that the number of signals impinging on the array be known or estimated.

- Limited angular resolution: for both algorithms the DOA estimation error increase as the angular separation of the signals becomes small.

- High computational complexity: algorithms require subspace decomposition techniques

To confirm these results we integrated the MUSIC algorithm in UTRA-TDD link-level simulation chain. The simulated scenario consists of 2- path signals of equal mean power that arrive at 8elemment uniformly spaced array at angles $80^{\circ}$ and $100^{\circ}$. In Figure 8 we can see that the MUSIC algorithm can only resolve two signals separated by $20^{\circ}$, if the Eb/N0 is higher than $20 \mathrm{~dB}$.

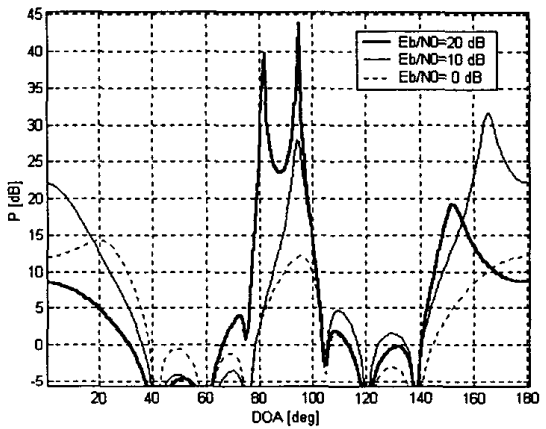

Fig.8. Typical MUSIC spatial spectrum, 2 paths with equal power, $80^{\circ}$ and $100^{\circ}, \mathrm{SF}=16$.

\section{B. Maximum Likelihood technique with SMET}

Maximum likelihood techniques were some of the first techniques to be investigated for DOA estimation. Since ML techniques were computationally intensive they were less popular than suboptimal subspace techniques. However, in terms of performance, the ML techniques are superior, especially in low signal-to-noise ratio conditions, typical of WCDMA systems.

In case of radio channel with $L$ multipath components and considering $K$ users, the number of paths needed to be resolved can be quite large $(K \times L)$. Straightforward application of ML estimation principles implies that log-likelihood function must be maximized with respect to the unknown parameters (amplitude, phase and DOA), for each multipath component. This results in a non-linear, $K \times L \times 3$-dimensional, maximization problem, which is computationally very intensive. To overcome this limitation, we implemented a moderate complexity ML DOA estimation based on the SMET scalar channel estimator. The basic structure is shown in Figure 9; it consists in applying the SMET to each of the $M$ array elements, and then estimate the DOA using the ML criterion based on the phase shift relation existing between array 
elements. This algorithm is of course no longer the optimum joint parameter estimator but it brings down the $K \times L \times 3$-dimensional maximization problem to $K \times L$ successive 3 -dimensional problems, thus reducing the computational complexity. The mathematical formulation of ML criterion for DOA estimation, using a Uniform Circular Array, can be see in [2].

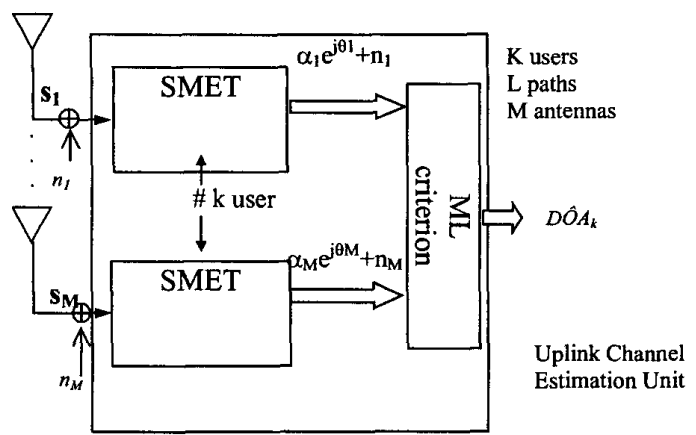

Fig.9. ML Estimation Unit based in M-SMET Estimation Units.

This technique does not make any assumption about the angular separation between the DOAs of the different paths and thus, the angular resolution is $0^{\circ}$. However a problem arises because the performance of the ML DOA estimation technique depend heavily on the quality of the scalar channel estimation provided by SMET. Therefore, when the threshold process is used within SMET to eliminate one path, we propose that the DOA estimation for current burst to keep the DOA estimation obtained in the previous burst. This technique assumes constant time-invariant DOAs across some bursts, this is a reasonable assumption which have been confirmed by numerous measurement campaigns [10]. To evaluate the ML-DOA channel estimation performance a single user were considered with the channel being modeled by a two-path with uncorrelated Rayleigh fading. The average power of the second path is $1 \mathrm{~dB}$ below the first path and the relative delay $1 \mathrm{Tc}$. The angular spread considered between the two paths was 0 . An UCA with $M=8$ antenna elements was used. The ML-DOA estimation performance was then examined in the UTRATDD link-level simulation chain, implemented according to current 3GPP specifications. Table 1 shows the rms error for the DOA (over 400 UMTS bursts) for different $E_{b} / N_{0}$ values. In the last column of Table 1 we can see the mean time of DOA stationary value required in number of UMTS bursts.

\section{CONCLUSIONS}

In this paper, we have proposed a ML approach to highresolution channel estimation in a UTRA-TDD system. As the main conclusions, we can point out:

- The proposed SMET algorithm clearly outperforms the 3GPP scalar channel estimator. A time resolution between consecutive paths equal to $1 \mathrm{Tc}$ could be achieved. A threshold operation was also introduced to select only the paths with enough SINR.

- An extension for antenna arrays of the SMET estimation algorithm, based on a ML criterion was proposed, and its performance assessed. The results have shown that the DOA accuracy estimation of the proposed ML algorithm is greater than the MUSIC algorithm. Furthermore it has been verified that the proposed ML-DOA estimation algorithm guarantees a DOA rms-error below $3^{\circ}$, even for multipath components with temporal spacing between consecutive paths equal to chip time interval, and $0^{\circ}$ of angular separation.

- Due the threshold processing the ML-DOA estimation algorithm assumes constant timeinvariant DOAs across some bursts, specially for low values of $E_{b} / N_{0}$.

Table. 1 : rms error for ML-DOA estimation using $M=8$ and SMET with $\mathrm{p}=1 \mathrm{E}-4$, 2-Rayleigh paths, $\Delta t=1 \mathrm{Tc}$, single user

\begin{tabular}{|c|c|c|c|}
\hline $\begin{array}{c}E_{b} / N_{0} \\
d B\end{array}$ & $\begin{array}{c}\text { rms_error_DOA } \\
\text { path1 [deg] }\end{array}$ & $\begin{array}{c}\text { rms_error_DOA } \\
\text { path2 [deg] }\end{array}$ & $\begin{array}{c}\text { Stationary } \\
\text { DOA in bursts }\end{array}$ \\
\hline 0 & 2,3 & 1,8 & 11 \\
\hline 4 & 2,1 & 1,6 & 9 \\
\hline 8 & 1,8 & 1,2 & 5 \\
\hline 12 & 1,7 & 1,1 & 2 \\
\hline
\end{tabular}

\section{REFERENCES}

[1] 3GPP TS 25.221, "Physical channels and mapping of transport channels onto physical channels (TDD)".

[2] P. Marques, A. Gameiro and J. Fernandes, "Vectorial Multipath Channel Estimation for the UTRA-TDD mode", IEEE Vehicular Tech. Conf, Atlantic City, Oct., 2001.

[3] J. C. Liberti, T. S. Rappaport, Smart Antennas for Wireless Communications: IS-95 and Third Generation CDMA Applications, Prentice Hall, 1999.

[4] ETSI, "Universal Mobile Telecommunications System, Selection procedures for the choice of radio transmission technologies of the UMTS", TR 101112, 1998, pp.42-43.

[5] P. Patel and J. Holtzmann, "Analysis of a simple successive interference cancellation scheme in DS/CDMA systems", IEEE J. Selec. Areas Commun., June 1994.

[6] 3GPP TS 25.224, "Physical Layer Procedures (TDD)".

[7] K. Higuichi, H. Andoh, "Expermental Evaluation of Combined Effect of Coherent Rake Combining and SIRBasead Fast TPC for Reverse Link of DS-CDMA Mobile Radio", IEEE J. Selec. Areas Commun., August 2000.

[8] 3GPP TS 25.201, "Physical Layer-General Description".

[9] J. Jeong, K. Sakaguchi, "Performance Analysis of MUSIC and ESPRIT Using Extended Array Mode Vector in Muliple Scattering Environment", WPMC' 01, Alborg, Denmark, Sept. 2001.

[10] L.M. Correia (Editor), "Wireless Flexible Personalised Communications", John Wiley \& Sons, 2001. 